\title{
Structure of the sets of mutually unbiased bases with cyclic symmetry
}

Ulrich Seyfarth, ${ }^{1}$ Luis L. Sánchez-Soto, ${ }^{1,2,3}$ and Gerd Leuchs ${ }^{1,2}$

1) Max-Planck-Institut für die Physik des Lichts, Günther-Scharowsky-Straße 1, Bau 24, 91058 Erlangen, Germany

${ }^{2)}$ Department für Physik, Universität Erlangen-Nürnberg, Staudtstraße \%, Bau 2, 91058 Erlangen, Germany

3) Departamento de Óptica, Facultad de Física, Universidad Complutense, 28040 Madrid, Spain

(Dated: 18 March 2021)

Mutually unbiased bases that can be cyclically generated by a single unitary operator are of special interest, since they can be readily implemented in practice. We show that, for a system of qubits, finding such a generator can be cast as the problem of finding a symmetric matrix over the field $\mathbb{F}_{2}$ equipped with an irreducible characteristic polynomial of a given Fibonacci index. The entanglement structure of the resulting complete sets is determined by two additive matrices of the same size. 


\section{INTRODUCTION}

Complementarity distinguishes the world of quantum phenomena from the realm of classical physics $\frac{1}{1}$. At a fundamental level, mutually unbiased bases (MUBs) provide, perhaps, the most accurate statement of complementarity. This notion emerged in the seminal work of Schwinger $\underline{2}^{-\underline{4}}$ and has gradually turned into a cornerstone of quantum information (see Ref. 5 for a comprehensive review). MUBs have long been known to provide an optimal scheme for quantum tomography $\underline{\underline{6} .7}$ and are central to the formulation of the discrete Wigner function $\underline{\underline{8}} \underline{-12}$. They have also been used in cryptographic protocols $\underline{13}-\underline{18}$, in quantum error correction codes $\underline{\underline{19}} \underline{\underline{23}}$, and in quantum game theory, in particular to provide a solution to the mean king problem $\underline{24} \underline{\underline{33}}$.

For a $d$-dimensional quantum system, it has been shown that the number of MUBs is at most $d+1 \underline{34}$. Actually, such a complete set of MUBs exists whenever $d$ is prime or power of

prime ${ }^{35}$. Remarkably though, there is no known answer for any other values of $d$, although there are some attempts to find a solution to this problem in some simple cases, such as $d=$ $6 \underline{36}-\underline{41}$ or when $d$ is a nonprime integer squared $\underline{\underline{42}} \underline{\underline{43}}$. Recent work suggests that the answer to this question may well be related with the non-existence of finite projective planes of certain orders $44 \underline{46}$ or with the problem of mutually orthogonal Latin squares in combinatorics $47-\underline{50}$. Furthermore, MUBs have interesting connections to symmetric informationally complete positive-operator-valued measures ${ }^{51}$ and complex $t$-designs $s^{52,53}$.

Many explicit constructions of MUBs in prime power dimensions have been proposed $\underline{\underline{54}}-\underline{59}$. However, irrespective of the approach, one has to face an intriguing question: different complete sets of MUBs exist with distinct entanglement properties $\underline{\underline{60}} \underline{\underline{66}}$. For the experimentalist, this information is of utmost importance, because the complexity of implementing a given set greatly depends on how many registers need to be entangled. Note carefully that this entanglement structure is different from the inequivalence of different sets of MUBs $\underline{67}, \underline{68}$.

In even prime-power dimensions, complete sets of MUBs can be shaped in a cyclic manner, as the multiples of a single generating basis $s^{\underline{60}} \underline{\underline{73}}$. This procedure rests on the properties of the so-called Fibonacci polynomials ${ }^{\underline{74}}$ and leads directly to quantum circuits that can be used for a simple practical realization of these bases.

In this work, we present a method to setup sets of cyclic MUBs with different entanglement structures. This is accomplished by unveiling certain structures within the different 
sets, which are related either to a field, an additive group or an additive semigroup. The key idea is a two-step generalization that softens the properties of the starting field structure, while preserving the proper features inherited from the Fibonacci polynomials.

This paper is organized as follows: In Section II we introduce the basic tools and definitions needed for the rest of our exposé. The main results are covered in Section III. which starts with complete sets possessing a field structure and discusses their extension to sets with a group and a semigroup structure. The generality of the method is confirmed in Section IV] whereas our conclusions are summarized in Section D.

\section{PRELIMINARIES}

\section{A. Mutually unbiased bases}

Definition II.1 (Mutually unbiased bases).

Let $\mathcal{H}=\mathbb{C}^{d}$ be a $d$-dimensional complex Hilbert space. A pair of orthonormal bases $\mathcal{B}_{j}=$ $\left\{\left|\psi_{\ell}^{(j)}\right\rangle\right\}$ and $\mathcal{B}_{j^{\prime}}=\left\{\left|\psi_{\ell^{\prime}}^{\left(j^{\prime}\right)}\right\rangle\right\}$ (with $j \neq j^{\prime}$ ) is said mutually unbiased if

$$
\left|\left\langle\psi_{\ell}^{(j)} \mid \psi_{\ell^{\prime}}^{\left(j^{\prime}\right)}\right\rangle\right|^{2}=\frac{1}{d}
$$

for all $\ell, \ell^{\prime} \in\{1, \ldots, d\}$.

In physical terms, this means that if the system is prepared in a state of the first basis, then all outcomes are equally probable when we conduct a measurement that probes the states of the second basis. Familiar examples are the spin states of a spin-1/2 particle for two perpendicular directions or any basis and its Fourier transform for any dimension $d$.

When the bases in the orthonormal set $\mathfrak{S}=\left\{\mathcal{B}_{0}, \ldots, \mathcal{B}_{r}\right\}$, with $r \in \mathbb{N}_{0}$, are pairwise unbiased, we say that $\mathfrak{S}$ is a set of MUBs. When such a set contains the maximal number of elements, $d+1$, it is called a complete set of MUBs.

As heralded in the introduction, for even prime-power dimensions, complete sets of MUBs can be constructed in a cyclic way. To understand this point, we observe that any basis in $\mathbb{C}^{d}$ can be always identified with a unitary matrix $U \in M_{d}(\mathbb{C})\left(M_{d}(\mathbb{C})\right.$ stands for the $d \times d$ matrices over $\mathbb{C}$ ) acting on this space, since the columns of $U$ define an orthonormal basis and vice versa. Since $U^{2}, U^{3}, \ldots$ are unitary, they also define bases. 
Definition II.2 (Cyclic mutually unbiased bases).

A complete set $\mathfrak{S}=\left\{\mathcal{B}_{0}, \ldots, \mathcal{B}_{d}\right\}$ of MUBs is called cyclic, if there exists a unitary matrix $U \in M_{d}(\mathbb{C})$, such that the columns of $U, U^{2}, U^{3}, \ldots, U^{d+1}=\mathbb{1}_{d}$ coincide with the bases in $\mathfrak{s .}$

To classify different sets of MUBs, the notion of equivalence has to be established.

Definition II.3 (Equivalence of complete set of mutually unbiased bases).

Let $\mathfrak{S}=\left\{B_{0}, \ldots, B_{d}\right\}$ and $\mathfrak{S}^{\prime}=\left\{B_{0}^{\prime}, \ldots, B_{d}^{\prime}\right\}$ be two complete sets of MUBs. Both sets are said to be equivalent if there holds

$$
\mathcal{B}_{j}^{\prime}=U \mathcal{B}_{\pi(j)} W_{j}
$$

for any unitary matrix $U \in M_{d}(\mathbb{C})$, a permutation $\pi$ of $\{0, \ldots, d\}$ and monomial matrices $W_{j}$ with $j \in\{0, \ldots, d\}$.

We recall that a matrix $W$ is called monomial, if it can be expressed as $W=D \Pi$, with $D=\operatorname{diag}\left(\lambda_{1}, \ldots, \lambda_{d}\right)$ is diagonal and $\Pi$ is a permutation. We assume $\left|\lambda_{i}\right|=1$, with $i \in\{1, \ldots, d\}$; i.e., $W$ is unitary.

\section{B. Pauli operators and entanglement structure}

Let us consider a prime-power dimension $d=p^{m}$, with $p$ prime and $m \in \mathbb{N}$. On the Hilbert space $\mathcal{H}=\mathbb{C}^{p}$, with canonical orthonormal basis $\left\{|i\rangle: i \in \mathbb{F}_{p}\right\}$ we define the Pauli operators by

$$
Z|i\rangle=\omega^{i}|i\rangle, \quad X|i\rangle=|i \oplus 1\rangle,
$$

where $\omega=\exp (2 \pi \mathrm{i} / d)$ is the first $p$-th root of unity and the addition $\oplus$ must be understood $\bmod p$.

This concept can be generalized to the Hilbert space $\mathcal{H}=\mathbb{C}^{d}$ by introducing the $2 m$ dimensional vector $\vec{a}=\left(a_{1}^{z}, \ldots, a_{m}^{z} ; a_{1}^{x}, \ldots, a_{m}^{x}\right)^{t} \in \mathbb{F}_{p}^{2 m}$, and in terms of it, the set

$$
Z X(\vec{a})= \begin{cases}(-\mathrm{i})^{a_{1}^{z} a_{1}^{x}} Z^{a_{1}^{z}} X^{a_{1}^{x}} \otimes \cdots \otimes(-\mathrm{i})^{a_{m}^{z} a_{m}^{x}} Z^{a_{m}^{z}} X^{a_{m}^{x}}, & p=2 \\ Z^{a_{1}^{z}} X^{a_{1}^{x}} \otimes \cdots \otimes Z^{a_{m}^{z}} X^{a_{m}^{x}}, & p \neq 2\end{cases}
$$


The set of these Pauli operators is just the Weyl-Heisenberg group factorized by its center. Two Pauli operators defined by the vectors $\vec{a}, \vec{b} \in \mathbb{F}_{p}^{2 m}$ obey the symplectic commutation relation

$$
Z X(\vec{a}) \cdot Z X(\vec{b})=\omega^{(\vec{a}, \vec{b})} Z X(\vec{b}) \cdot Z X(\vec{a})
$$

with the symplectic inner product defined as

$$
(\vec{a}, \vec{b})=\sum_{k=1}^{m} a_{k}^{z} b_{k}^{x}-a_{k}^{x} b_{k}^{z} .
$$

According with the ideas in Ref. 54, complete sets of MUBs arise straightforwardly from a partition of the set of Pauli operators into $d+1$ subsets of $d-1$ commuting operators, called classes. These classes $\mathfrak{C}_{j}^{\prime \prime}$ are given for $j \in\{0, \ldots, d\}$ by

$$
\mathfrak{C}_{j}^{\prime}=\mathfrak{C}_{j} \cup\left\{\mathbb{1}_{d}\right\}=\left\{Z X(\vec{a}): \vec{a}=G_{j} \vec{c}: \vec{c} \in \mathbb{F}_{p}^{m}\right\}
$$

in terms of the generators $G_{j}$.

The Pauli operators within a class $\mathfrak{C}_{j}$ commute by construction. But, we can check if the Pauli operators corresponding to different subsystems also commute. If so, the property measured by the corresponding basis will be a property for which this subsystem is not entangled with the rest of the system. In principle, all possible partitions of the number of subsystems $m \in \mathbb{N}$ are possible, from completely factorizable to fully entangled systems. Finally, any set of basis can be classified by a vector $\vec{n}$, where each element counts the number of bases having a specific entanglement structure. The length of $\vec{n}$ is given by the number of partitions of $m$, and the first entry gives the number of completely factorizable bases.

\section{Fibonacci polynomials}

Our analysis of complete sets of cyclic MUBs will rely on the properties of Fibonacci polynomials, which are a generalization of the well-known Fibonacci sequence.

Definition II.4 (Fibonacci polynomials).

Over an arbitrary field $K$, we define the Fibonacci polynomials $F_{n}(x)\left(n \in \mathbb{N}_{0}\right)$ by the recursion relation

$$
F_{j+1}(x)=x F_{j}(x)+F_{j-1}(x)
$$


with $F_{0}=0$ and $F_{1}=1$.

In our context, we exclusively deal with the ground field $\mathbb{F}_{2}$ and possibly its extensions. As the Fibonacci sequence, also the Fibonacci polynomials can be constructed using a generator, namely,

$$
\mathcal{A}=\left(\begin{array}{ll}
x & 1 \\
1 & 0
\end{array}\right) \equiv\left(\begin{array}{ll}
F_{2}(x) & F_{1}(x) \\
F_{1}(x) & F_{0}(x)
\end{array}\right),
$$

so that the powers of this generator are

$$
\mathcal{A}^{j}=\left(\begin{array}{cc}
F_{j+1}(x) & F_{j}(x) \\
F_{j}(x) & F_{j-1}(x)
\end{array}\right) .
$$

One can verify that

$$
F_{j}(x) F_{k+1}(x)+F_{j-1}(x) F_{k}(x)=F_{k+1}(x) .
$$

The Fibonacci numbers satisfy several well-known divisibility relations; we will need their counterparts for Fibonacci polynomials over $K=\mathbb{F}_{2}$.

Definition II.5 (Fibonacci index).

The Fibonacci index of an irreducible polynomial $p \in K[x]$ is defined as the minimum number $n \in \mathbb{N}$, such that $p(x)$ divides $F_{n}(x)$.

If the Fibonacci polynomials are defined over $\mathbb{F}_{2}$, the Fibonacci index of any irreducible polynomial is either a divisor of $2^{m}-1$ or $2^{m}+1$, with $m$ being the degree of $p(x)^{75}$.

\section{METHOD}

The aim of this article is to investigate a method to generate complete sets of cyclic MUBs with different entanglement structures. According to intrinsic structures, three different constructions will be discussed: we start with a basic method and present then two consecutive generalizations.

\section{A. Field-based sets}

In Ref. 73 it has been shown how to construct complete sets of cyclic MUBs in dimension $d=2^{m}, m \in \mathbb{N}$. This problem can accordingly be reduced to finding a suitable symplectic 
stabilizer matrix $C \in M_{2 m}\left(\mathbb{F}_{2}\right)^{71}$. In fact, such a matrix can be written as

$$
C=\left(\begin{array}{cc}
B & \mathbb{1}_{m} \\
\mathbb{1}_{m} & 0_{m}
\end{array}\right)
$$

where $B$ is a symmetric and invertible matrix whose characteristic polynomial has Fibonacci index $d+1$. Each solution leads to a complete set of cyclic MUBs.

It is worth noticing that the powers of $C$ can be easily written, according to Eq. (10), as

$$
C^{j}=\left(\begin{array}{cc}
F_{j+1}(B) & F_{j}(B) \\
F_{j}(B) & F_{j-1}(B)
\end{array}\right)
$$

The generators of the cyclic sets can be written as

$$
G_{j}=C^{j} G_{0}, \quad G_{0}=\left(\begin{array}{c}
\mathbb{1}_{m} \\
0_{m}
\end{array}\right)
$$

so, using (13), we have

$$
G_{j}=\left(\begin{array}{c}
F_{j+1}(B) \\
F_{j}(B)
\end{array}\right)
$$

For our purposes in what follows, it will prove convenient to rewrite these generators in a standard form as

$$
\bar{G}_{j}=\left(\begin{array}{c}
F_{j+1}(B) F_{j}^{-1}(B) \\
\mathbb{1}_{m}
\end{array}\right),
$$

for $j \in\{1, \ldots, d\}$ and $\bar{G}_{0} \equiv G_{0}$. This transformation is possible by exploiting the properties of the generation of the classes in (7), where each invertible square matrix multiplied from the left to all elements $\vec{c}$ is only permuting these elements, thus multiplying any generator matrix $G_{j}$ from the right by an invertible square matrix of appropriate size does not change the generated set.

Lemma III.1 (Generators are finite field representation). The set of $m \times m$ upper submatrices of the generators $\left\{G_{j}\right\}$ of a complete set of cyclic MUBs in the standard form (16) is a representation of the finite field $\mathbb{F}_{2^{m}}$. 
Proof. For a complete set of cyclic MUBs, all the generators $\bar{G}_{j}$ have to be distinct for $j \in$ $\{1, \ldots, d\}$. The matrices $F_{j+1}(B) F_{j}^{-1}(B)=p_{j}(B)$ are polynomials that can be taken modulo the characteristic polynomial of $B$. This is irreducible and has order $m$. As there are $d=2^{m}$ different generators, each element of the field can be represented, with $p_{j}(B)=0_{m}$ being the neutral element of addition and $p_{d / 2}(B)=\mathbb{1}_{m}$ the neutral element of multiplication.

Lemma III.1 justifies that we call these sets field-based sets. All sets exhibiting this structure, will have three bases (and therefore three corresponding generators) with an entanglement structure that is completely factorizable, namely

$$
G_{0}=\left(\begin{array}{c}
\mathbb{1}_{m} \\
0_{m}
\end{array}\right), \quad \bar{G}_{d / 2}=\left(\begin{array}{c}
\mathbb{1}_{m} \\
\mathbb{1}_{m}
\end{array}\right), \quad G_{d}=\left(\begin{array}{c}
0_{m} \\
\mathbb{1}_{m}
\end{array}\right),
$$

where the classes make the sets of all Pauli $Z, Y$, and $X$ operators, respectively. Therefore, to change the number of completely factorizable bases within a set, we need to adapt the form of the stabilizer matrix $C$ in (12). In the following, we will show how to build complete sets of MUBs with only two and one completely factorizable bases by two generalizations. For this, we start from an approach for the generators in standard form and try to calculate the corresponding stabilizer matrix.

\section{B. Group-based sets}

To reduce the number of completely factorizable bases, we break down the field structure discussed in the last section to an additive group structure. This excludes the neutral element of the multiplication, as it accounts for one of the completely factorizable bases.

If, in the standard form, the new generators are

$$
\bar{G}_{j}=\left(\begin{array}{c}
F_{j+1}(B) F_{j}^{-1}(B) R \\
\mathbb{1}_{m}
\end{array}\right), \quad G_{0}=\left(\begin{array}{c}
\mathbb{1}_{m} \\
0_{m}
\end{array}\right)
$$

Theorem 4.4 of $^{54}$ would still be fulfilled, as long as $R \in M_{m}\left(\mathbb{F}_{2}\right)$ is invertible. This leads to a stabilizer matrix

$$
C=\left(\begin{array}{cc}
B & R \\
R^{-1} & 0_{m}
\end{array}\right)
$$


or, in terms of Fibonacci polynomials,

$$
C^{j}=\left(\begin{array}{cc}
F_{j+1}(B) & F_{j}(B) R \\
R^{-1} F_{j}(B) & R^{-1} F_{j-1}(B) R
\end{array}\right) .
$$

By setting $R=\mathbb{1}_{m}$, it is obvious that this stabilizer is a generalization of the one used for the field-based sets. From Theorem 4.4 of Ref. 54 all matrices $p_{j}(B) R$ have to be symmetric. Furthermore, an analogous argument as for the field-based sets leads to the condition, that the characteristic polynomial of $B$ should have Fibonacci index $d+1$. By the following lemma we can substantially reduce one of the conditions.

Lemma III.2 (Symmetrizer applies to polynomials).

Let $K$ denote an arbitrary field. Let us assume given the invertible matrix $B \in M_{m}(K)$, there exists an invertible and symmetric symmetrizer $R \in M_{m}(K)$ such that $B R$ is symmetric. Then, all polynomials of the form $p(B) R$, with $p(B) \in K[x]$, are also symmetric.

Proof. If $R$ and $B R$ are symmetric, then for any matrix $B^{k} R$ with $k \in \mathbb{N}$ we have

$$
\left(B^{k} R\right)^{t}=R^{t} B^{t}\left(B^{k-1}\right)^{t}=B R^{t} B^{t}\left(B^{k-2}\right)^{t}=\ldots=B^{k} R .
$$

As sums of symmetric matrices are symmetric, this holds for all polynomials $p(B)$. Considering the cases $k=0$ and $k=1$, the converse statement is obvious.

This Lemma and the previous discussion lead to the following final set of conditions to be fulfilled to construct a complete set of cyclic MUBs with three or two completely factorizable bases for invertible $R, B \in M_{m}\left(\mathbb{F}_{2}\right)$ :

(i) $R$ and $B R$ are symmetric.

(ii) The characteristic polynomial of $B$ has Fibonacci index $d+1$.

For each valid $B$ a corresponding symmetrizer matrix $R$ exists which is additionally a square root of unity $\underline{76}$. An algorithm can be found in Ref. 77 and 78 .

To construct only sets which have not more than two completely factorizable bases, $R$ has to destroy the field structure as will be seen by the following lemma.

Lemma III.3 (Destruction of field structure).

Iff the symmetric matrix $R \in M_{m}\left(\mathbb{F}_{2}\right)$ does not equal any polynomial of $B \in M_{m}\left(\mathbb{F}_{2}\right)$, where $R$ and $B$ are chosen according to the conditions of group-based sets, the resulting complete set of cyclic MUBs has exactly two completely factorizable bases. 
Proof. If $R$ is chosen to be a polynomial of $B$, say $q(B)$, the generators $\bar{G}_{j}$ in (18) will read

$$
\bar{G}_{j}=\left(\begin{array}{c}
p_{j}(B) q(B) \\
\mathbb{1}_{m}
\end{array}\right), \quad G_{0}=\left(\begin{array}{c}
\mathbb{1}_{m} \\
0_{m}
\end{array}\right)
$$

and thus the classes will be a permuted version of the field-based sets given in (16). Conversely, as soon as $R$ does not equal any polynomial of $B$, none of the generators will equal $\left(\mathbb{1}_{m}, \mathbb{1}_{m}\right)^{t}$, thus defining the set of Pauli $Y$ operators. But any generator in the form $\left(p(B) R, \mathbb{1}_{m}\right)^{t}$ will have off-diagonal entries in $p(B) R$ for $R \neq \mathbb{1}_{m} \equiv B^{0}$ and $p(B) \neq 0_{m}$ being a non-zero polynomial of $B$. Therefore, none of the generators can produce a completely factorizable basis.

It is obvious, that all matrices $R^{\prime}$ lead to a set with the same entanglement properties as a set with $R$, as long as $R^{\prime}=q(B) R$ holds for any non-zero polynomial $q$ of $B$, as it permutes only the set of bases.

\section{Semigroup-based sets}

The second generalization is to further restrict the additive group to an additive semigroup structure, which additionally excludes from the set the neutral element of addition. This is achieved by adding a symmetric matrix $A \in M_{m}\left(\mathbb{F}_{2}\right)$ to the matrices $F_{j+1}(B)\left(F_{j}(B)\right)^{-1} R$ with $j \in\{1, \ldots, d\}$ in order to preserve Theorem 4.4 in Ref. 54. We will call these sets semigroup-based sets.

In standard form, the generators look like

$$
\bar{G}_{j}=\left(\begin{array}{c}
F_{j+1}(B)\left(F_{j}(B)\right)^{-1} R+A \\
\mathbb{1}_{m}
\end{array}\right), \quad G_{0}=\left(\begin{array}{c}
\mathbb{1}_{m} \\
0_{m}
\end{array}\right)
$$

To find the corresponding stabilizer matrix $C$, we note that these generators correspond to those given by the product of powers of $C$ with $G_{0}$ and

$$
G_{j}=\left(\begin{array}{c}
F_{j+1}(B)+A R^{-1} F_{j}(B) \\
R^{-1} F_{j}(B)
\end{array}\right)
$$

As $C G_{0}=G_{1}$ should hold, the first column of $C$ has to be equal to $G_{1}$. So we can approach $C$ as $C(x, y)$ with the two free parameters $x, y \in M_{m}\left(\mathbb{F}_{2}\right)$ and fix them by applying $C G_{1}=G_{2}$ 
as

$$
\left(\begin{array}{cc}
B+A R^{-1} & x \\
R^{-1} & y
\end{array}\right)\left(\begin{array}{c}
B+A R^{-1} \\
R^{-1}
\end{array}\right)=\left(\begin{array}{c}
B^{2}+B A R^{-1}+A R^{-1} B+A R^{-1} A R^{-1}+x R^{-1} \\
R^{-1} B+R^{-1} A R^{-1}+y R^{-1}
\end{array}\right),
$$

so that

$$
G_{2}=\left(\begin{array}{c}
B^{2}+1+A R^{-1} B \\
R^{-1} B
\end{array}\right)
$$

Solving these equations we find a new form of the stabilizer matrix, namely

$$
C=\left(\begin{array}{cc}
B+A R^{-1} & R+B A+A R^{-1} A \\
R^{-1} & R^{-1} A
\end{array}\right) .
$$

Setting $A=0$ leads to (19), confirming that we have found a further generalization of the group-based construction. As a next step, it is helpful to calculate the powers of $C$, as we can easily check further properties of this matrix. We find once again a very compact form, in terms of the Fibonacci polynomials;

$$
C^{j}=\left(\begin{array}{cc}
F_{j+1}(B)+A R^{-1} F_{j}(B) & F_{j+1}(B) A+F_{j}(B) R+A R^{-1}\left[F_{j}(B) A+F_{j-1}(B) R\right] \\
R^{-1} F_{j}(B) & R^{-1}\left[F_{j}(B) A+F_{j-1}(B) R\right]
\end{array}\right) .
$$

Equation (28) allows us to check that $C^{d+1}=\mathbb{1}_{2 m}$. As long as the Fibonacci index of $B$ is $d+1$ as in the former constructions, $C^{j} \neq \mathbb{1}_{2 m}$ for any $j \in\{1, \ldots, d\}$ if one considers only the term $R^{-1} F_{j}(B)$. Finally, we can check if all powers of $C$ permute the generators as expected, namely by checking if $C^{j} G^{k}=G^{(j+k) \bmod (d+1)}$ :

$$
C^{j} G^{k}=\left(\begin{array}{c}
F_{j+1}(B) F_{k+1}(B)+F_{j}(B) F_{k}(B)+A R^{-1}\left[F_{j}(B) F_{k+1}(B)+F_{j-1}(B) F_{k}(B)\right] \\
R^{-1}\left[F_{j}(B) F_{k+1}(B)+F_{j-1}(B) F_{k}(B)\right]
\end{array}\right) .
$$

Using the general recursion relation (11) we find

$$
C^{j} G^{k}=\left(\begin{array}{c}
F_{k+j+1}(B) A R^{-1} F_{k+j}(B) \\
R^{-1} F_{k+j}(B)
\end{array}\right),
$$

which is the expected result.

The conditions to be fulfilled to construct a complete set of cyclic MUBs, using the stabilizer (27) for $A \in M_{m}\left(\mathbb{F}_{2}\right)$ and for invertible $R, B \in M_{m}\left(\mathbb{F}_{2}\right)$ are: 
(i) $A, R$, and $B R$ are symmetric.

(ii) The characteristic polynomial of $B$ has Fibonacci index $d+1$.

This generates complete sets of cyclic MUBs with three, two and one completely factorizable bases. To have only sets with one factorizable basis, we have to choose $R$ according to Lemma III.3 and add a similar condition which breaks down the additive group structure into an additive semigroup structure. Regarding (23), we have to guarantee that no generator of the form $\left(\mathbb{1}_{m}, \mathbb{1}_{m}\right)^{t}$ or $\left(0_{m}, \mathbb{1}_{m}\right)^{t}$ is produced. This additional condition is given by the following lemma.

Lemma III.4 (Destruction of additive group structure).

Iff the symmetric matrix $A \in M_{m}\left(\mathbb{F}_{2}\right)$ does not equal any of the matrices $p_{j}(B) R+D_{l}$, where $R$ and $B$ are chosen according to the conditions of semigroup-based sets, with $p_{j}(B)$ being the polynomials in $B$ and $j \in\{1, \ldots, d\}$, the resulting complete set of cyclic MUBs has exactly one completely factorizable basis. The matrix $D_{l}$ denotes the diagonal matrix for which $\left(d_{i i}\right)=l_{i}$ holds for $l=\left(l_{1}, \ldots, l_{m}\right), i \in\{1, \ldots, m\}$ and with $l_{i} \in 0,1$.

Proof. If $A$ is chosen to be a polynomial of $B$, say $q(B)$, multiplied by $R$, the class for which $q(B)=p_{j}(B)$ holds will produce the set of Pauli $X$ operators, therefore this possibility has to be excluded. Furthermore, if instead of $0_{m}$, a matrix $D_{l}$ with only diagonal entries

appears in the $m \times m$ upper submatrix of the generator which would then equal $\left(D_{l}, \mathbb{1}_{m}\right)^{t}$, for all cases of $D_{l}$ a completely factorizable basis would be created. As long as this is not the case, the off-diagonal parts of the $m \times m$ upper submatrix of the generators in standard form will lead to bases which are not completely factorizable.

\section{COMPLETENESS}

Finally, we have to guarantee that our method produces MUBs with all the possible entanglement structures within the same equivalence class (in the sense of Definition 【I.3) and which are allowed by the scheme provided by Bandyopadhyay et $a l^{54}$. Of course, this does not include sets which have no completely factorizable bases.

The definition of equivalence can be restated in terms of the class generators $G_{j}$ with $j \in\{0, \ldots, d\}$, an arbitrary symplectic matrix $f \in \operatorname{Sp}_{2 m}\left(\mathbb{F}_{2}\right)$ and a permutation $Q$ of the 
elements within a class and a permutation $\pi_{j}$ of the index set of the set of bases.$^{73}$ By (7), the permutation $Q$ is of no relevance. As long, as we consider only the set of generators $G_{j}$ and not their ordering, also the permutation $\pi_{j}$ becomes irrelevant. So, we are left with the symplectic matrix $f$ that, in general, would be given by

$$
f=\left(\begin{array}{ll}
s & t \\
u & v
\end{array}\right)
$$

where $s, t, u, v \in M_{m}\left(\mathbb{F}_{2}\right)$. We start with a lemma:

Lemma IV.1 (Equivalence of different sets).

The semigroup-based sets with the stabilizer matrix given by (27) are equivalent to the fieldbased sets with the stabilizer matrix given by (12).

Proof. For a certain choice of the symplectic matrix $f$ we should be able to convert fieldbased sets into semigroup-based sets. This can be accomplished by multiplying the generators by

$$
f=\left(\begin{array}{cc}
s & t \\
0_{m} & v
\end{array}\right)
$$

where $s, t, v \in M_{m}\left(\mathbb{F}_{2}\right)$ and gives

$$
f G_{0}=\left(\begin{array}{c}
s \\
0_{m}
\end{array}\right), \quad f \bar{G}_{j}=\left(\begin{array}{c}
s p_{j}(B) s^{-1} s^{t} s+t s^{t} \\
\mathbb{1}_{m}
\end{array}\right),
$$

where we can recognize $s p_{j}(B) s^{-1}$ as $p_{j}\left(B^{\prime}\right), s^{t} s=R$ and $t s^{t}=A$ if $s$ and $t$ are chosen accordingly. If we set $t=0_{m}$, also the group-based sets with the stabilizer matrix given by (19) belong to the same equivalence class.

Finally, we confirm the completeness of our scheme.

Theorem IV.2 (Completeness of the construction scheme).

The stabilizer matrix (27) leads to complete sets of MUBs with all the possible entanglement structures. Moreover, they are equivalent to the sets generated via the stabilizer matrix (12).

Proof. By Lemma IV.1 the diffferent sets are equivalent. To show that the construction captures all possible sets within this equivalence class and according to Theorem 4.4 of 
Ref. 54, we have to be able to apply an arbitrary symplectic matrix $f \in \operatorname{Sp}_{2 m}\left(\mathbb{F}_{2}\right)$ to the generators (23), so we get

$$
f G_{0}=\left(\begin{array}{l}
s \\
u
\end{array}\right), \quad f \bar{G}_{j}=\left(\begin{array}{c}
s p_{j}(B) R+s A+t \\
u p_{j}(B) R+u A+v
\end{array}\right)
$$

for

$$
f=\left(\begin{array}{ll}
s & t \\
u & v
\end{array}\right)
$$

with $s, t, u, v \in M_{m}\left(\mathbb{F}_{2}\right)$. We still are free to reset any of the generators to the $G_{0}$ by

$$
f^{\prime}=\left(\begin{array}{cc}
s^{\prime} & t^{\prime} \\
u^{\prime} & v^{\prime}
\end{array}\right)
$$

which gives

$$
f^{\prime} f G_{0}=\left(\begin{array}{c}
s^{\prime} s+t^{\prime} u \\
u^{\prime} s+v^{\prime} u
\end{array}\right)
$$

and leads to the condition that $u^{\prime} s+v^{\prime} u$. Applying $f^{\prime}$ to $f$ leads then to a matrix where the lower left block equals $0_{m}$, thus we can chose a symplectic matrix in the form (32), where $v \equiv\left(s^{t}\right)^{-1}$ as long as $f$ is symplectic. For the generators of the semigroup-based sets we get then the same result we found in Lemma IV.1.

\section{CONCLUSIONS}

We have shown how to generate complete sets of cyclic MUBs with different entanglement structures by taking advantage of the properties of the Fibonacci polynomials. Two levels of generalization raise the basic field-based sets with three completely factorizable bases to the group-based sets with two completely factorizable bases and finally to the semigroup-based sets with only one completely factorizable basis.

Finally, we have proven that in this way we can generate all the possible entanglement structures. Of course, sets with zero factorizable bases are out of scope, as the standard basis cannot be part of the set. In principle, this should be realizable by generalizing again the discussed method. 


\section{ACKNOWLEDGMENTS}

Financial support from the EU FP7 (Grant Q-ESSENCE) and the Spanish DGI (Grant FIS2011-26786) is gratefully acknowledged.

\section{REFERENCES}

${ }^{1}$ N. Bohr, "Das Quantenpostulat und die neuere Entwicklung der Atomistik," Naturwissenschaften 16, 245-248 (1928).

2 J. Schwinger, "Unitary operator basis," Proc. Natl. Acad. Sci. USA 46, 570-576 (1960).

${ }^{3}$ J. Schwinger, "Unitary transformations and the action principle," Proc. Natl. Acad. Sci. USA 46, 883-897 (1960).

${ }^{4}$ J. Schwinger, "The special canonical group," Proc. Natl. Acad. Sci. USA 46, 1401-1415 (1960).

${ }^{5}$ T. Durt, B.-G. Englert, I. Bengtsson, and K. Życzkowski, "On mutually unbiased bases," Int. J. Quantum Inf. 8, 535-640 (2010).

${ }^{6}$ W. K. Wootters, "Quantum mechanics without probability amplitudes," Found. Phys. 16, 391-405 (1986).

${ }^{7}$ W. K. Wootters and B. D. Fields, "Optimal state-determination by mutually unbiased measurements," Ann. Phys. 191, 363-381 (1989).

${ }^{8}$ W. K. Wootters, "A Wigner-function formulation of finite-state quantum mechanics," Ann. Phys. 176, 1-21 (1987).

${ }^{9}$ R. Asplund and G. Björk, "Reconstructing the discrete Wigner function and some properties of the measurement bases," Phys. Rev. A 64, 012106 (2001).

${ }^{10}$ K. S. Gibbons, M. J. Hoffman, and W. K. Wootters, "Discrete phase space based on finite fields," Phys. Rev. A 70, 062101 (2004).

${ }^{11}$ A. Vourdas, "Quantum systems with finite Hilbert space," Rep. Prog. Phys. 67, 267-320 (2004).

${ }^{12}$ G. Björk, A. B. Klimov, and L. L. Sánchez-Soto, "The discrete Wigner function," Prog. Opt. 51, 469-516 (2008).

${ }^{13}$ H. Bechmann-Pasquinucci and A. Peres, "Quantum cryptography with 3-state systems," Phys. Rev. Lett. 85, 3313-3316 (2000). 
${ }^{14}$ M. Bourennane, A. Karlsson, and G. Björk, "Quantum key distribution using multilevel encoding," Phys. Rev. A 64, 012306 (2001).

${ }^{15}$ G. M. Nikolopoulos and G. Alber, "Security bound of two-basis quantum-key-distribution protocols using qudits," Phys. Rev. A 72, 032320 (2005).

${ }^{16}$ I.-C. Yu, F.-L. Lin, and C.-Y. Huang, "Quantum secret sharing with multilevel mutually (un)biased bases," Phys. Rev. A 78, 012344 (2008).

${ }^{17}$ Z.-X. Xiong, H.-D. Shi, Y.-N. Wang, L. Jing, J. Lei, L.-Z. Mu, and H. Fan, "General quantum key distribution in higher dimension," Phys. Rev. A 85, 012334 (2012).

${ }^{18}$ M. Mafu, A. Dudley, S. Goyal, D. Giovannini, M. McLaren, M. J. Padgett, T. Konrad, F. Petruccione, N. Lütkenhaus, and A. Forbes, "Higher-dimensional orbital-angularmomentum-based quantum key distribution with mutually unbiased bases," Phys. Rev. A 88, 032305 (2013).

${ }^{19}$ D. Gottesman, "Class of quantum error-correcting codes saturating the quantum Hamming bound," Phys. Rev. A 54, 1862-1868 (1996).

${ }^{20}$ A. R. Calderbank and P. W. Shor, "Good quantum error-correcting codes exist," Phys. Rev. A 54, 1098-1105 (1996).

${ }^{21}$ A. R. Calderbank, E. M. Rains, P. W. Shor, and N. J. A. Sloane, "Quantum error correction and orthogonal geometry," Phys. Rev. Lett. 78, 405-408 (1997).

${ }^{22}$ R. B. Griffiths, "Channel kets, entangled states, and the location of quantum information," Phys. Rev. A 71, 042337 (2005).

${ }^{23}$ M. Pawłowski and M. Żukowski, "Entanglement-assisted random access codes," Phys. Rev. A 81, $042326(2010)$.

${ }^{24}$ Y. Aharonov and B.-G. Englert, "The mean king's problem: spin 1," Z. Naturforsch. 56a, 16-19 (2001).

${ }^{25}$ B.-G. Englert and Y. Aharonov, "The mean king's problem: Prime degrees of freedom," Phys. Lett. A 284, 1-5 (2001).

${ }^{26}$ P. K. Aravind, "Solution to the king's problem in prime power dimensions," Z. Naturforsch. 58a, 85-92 (2003).

${ }^{27}$ A. Klappenecker and M. Rötteler, "New tales of the mean king," arXiv: quant-ph/0502138 (2005).

${ }^{28}$ A. Hayashi, M. Horibe, and T. Hashimoto, "Mean king's problem with mutually unbiased bases and orthogonal Latin squares," Phys. Rev. A 71, 052331 (2005). 
${ }^{29}$ J. P. Paz, A. J. Roncaglia, and M. Saraceno, "Qubits in phase space: Wigner-function approach to quantum-error correction and the mean-king problem," Phys. Rev. A 72, 012309 (2005).

${ }^{30}$ T. Durt, "About Weyl and Wigner tomography in finite-dimensional Hilbert spaces," Open Sys. Inf. Dyn. 13, 403-413 (2006).

${ }^{31}$ G. Kimura, H. Tanaka, and M. Ozawa, "Solution to the mean king's problem with mutually unbiased bases for arbitrary levels," Phys. Rev. A 73, 050301(R) (2006).

${ }^{32}$ A. Kalev, A. Mann, and M. Revzen, "Quantum-mechanical retrodiction through an extended mean king problem," EPL 104, 50008 (2013).

${ }^{33}$ M. Revzen, "Maximal entanglement, collective coordinates and tracking the king," J. Phys. A 46, 075303 (2013).

${ }^{34}$ I. D. Ivanovic, "Geometrical description of quantal state determination," J. Phys. A 14, 3241-3245 (1981).

${ }^{35}$ A. R. Calderbank, P. J. Cameron, W. M. Kantor, and J. J. Seidel, " $\mathbb{Z}_{4}$-kerdock codes, orthogonal spreads, and extremal Euclidean line-sets," Proc. London Math. Soc. 75, 436480 (1997).

${ }^{36}$ M. Grassl, "On SIC-POVMs and MUBs in dimension 6," arXiv: quant-ph/0406175 (2004).

${ }^{37} \mathrm{P}$. Butterley and W. Hall, "Numerical evidence for the maximum number of mutually unbiased bases in dimension six," Phys. Lett. A 369, 5-8 (2007).

${ }^{38} \mathrm{~S}$. Brierley and S. Weigert, "Maximal sets of mutually unbiased quantum states in dimension 6," Phys. Rev. A 78, 042312 (2008).

${ }^{39}$ S. Brierley and S. Weigert, "Constructing mutually unbiased bases in dimension six," Phys. Rev. A 79, 052316 (2009).

${ }^{40}$ P. Raynal, X. Lü, and B.-G. Englert, "Mutually unbiased bases in six dimensions: The four most distant bases," Phys. Rev. A 83, 062303 (2011).

${ }^{41}$ D. McNulty and S. Weigert, "All mutually unbiased product bases in dimension 6," J. Phys. A 45, 135307 (2012).

${ }^{42} \mathrm{C}$. Archer, "There is no generalization of known formulas for mutually unbiased bases," J. Math. Phys. 46, 022106 (2005).

${ }^{43} \mathrm{P}$. Wocjian and T. Beth, "New construction of mutually unbiased basis in square dimensions," Quantum Info. Compu. 5, 93-101 (2005).

${ }^{44}$ M. Saniga, M. Planat, and H. Rosu, "Mutually unbiased bases and finite projective 
planes," J. Opt. B 6, L19-L20 (2004).

${ }^{45}$ I. Bengtsson, "Mubs, polytopes and finite geometries," arXiv: quant-ph/0406174 (2004).

${ }^{46}$ S. Weigert and T. Durt, "Affine constellations without mutually unbiased counterparts," J. Phys. A 43, 402002 (2010).

${ }^{47}$ G. Zauner, "Quantum designs: Foundations of a noncommutative design theory," Int. J. Quantum Inf. 9, 445-507 (2011).

${ }^{48}$ W. K. Wootters, "Quantum measurements and finite geometry," Found. Phys. 36, 112-126 (2006).

${ }^{49}$ T. Paterek, B. Dakić, and Č. Brukner, "Mutually unbiased bases, orthogonal Latin squares, and hidden-variable models," Phys. Rev. A 79, 012109 (2009).

${ }^{50}$ J. L. Hall and A. Rao, "Mutually orthogonal Latin squares from the inner products of vectors in mutually unbiased bases," J. Phys. A 43, 135302 (2010).

${ }^{51}$ D. M. Appleby, "SIC-POVMs and MUBs: Geometrical relationships in prime dimension," in Foundations of Probability and Physics, AIP Conf. Proc., Vol. 5 (Amer. Inst. Phys., 2009) pp. $223-232$.

${ }^{52}$ A. Klappenecker and M. Rötteler, "Mutually unbiased bases are complex projective 2designs," in Proc. 2005 IEEE International Symposium on Information Theory (Adelaide, 2005) pp. 1740-1744.

${ }^{53}$ D. Gross, K. Audenaert, and J. Eisert, "Evenly distributed unitaries: On the structure of unitary designs," J. Math. Phys. 48, 052104 (2007).

${ }^{54}$ S. Bandyopadhyay, P. O. Boykin, V. Roychowdhury, and F. Vatan, "A new proof for the existence of mutually unbiased bases," Algorithmica 34, 512-528 (2002).

${ }^{55}$ K. R. Parthasarathy, "On estimating the state of a finite level quantum system," Infin. Dimens. Anal. Quantum Probab. Relat. Top. 7, 607-617 (2004).

${ }^{56}$ A. O. Pittenger and M. H. Rubin, "Mutually unbiased bases, generalized spin matrices and separability," Linear Algebra Appl. 390, 255-278 (2004).

${ }^{57}$ T. Durt, "About mutually unbiased bases in even and odd prime power dimensions," J. Phys. A 38, 5267-5284 (2005).

${ }^{58}$ A. B. Klimov, L. L. Sánchez-Soto, and H. de Guise, "Multicomplementary operators via finite Fourier transform," J. Phys. A 38, 2747-2760 (2005).

${ }^{59}$ M. R. Kibler and M. Planat, "A SU(2) recipe for mutually unbiased bases," Int. J. Mod. Phys. B 20, 1802-1807 (2006). 
${ }^{60}$ J. Lawrence, Č. Brukner, and A. Zeilinger, "Mutually unbiased binary observable sets on $n$ qubits," Phys. Rev. A 65, 032320 (2002).

${ }^{61}$ J. Lawrence, "Mutually unbiased bases and trinary operator sets for n qutrits," Phys. Rev. A 70, 012302 (2004).

${ }^{62}$ J. L. Romero, G. Björk, A. B. Klimov, and L. L. Sánchez-Soto, "Structure of the sets of mutually unbiased bases for $n$ qubits," Phys. Rev. A 72, 062310 (2005).

${ }^{63}$ A. Garcia, J. L. Romero, and A. B. Klimov, "Generation of bases with definite factorization for an $\mathrm{n}$-qubit system and mutually unbiased sets construction," J. Phys. A 43, 385301 (2010).

${ }^{64}$ M. Wieśniak, T. Paterek, and A. Zeilinger, "Entanglement in mutually unbiased bases," New J. Phys. 13, 053047 (2011).

${ }^{65}$ J. Řeháček, Z. Hradil, A. B. Klimov, G. Leuchs, and L. L. Sánchez-Soto, "Sizing up entanglement in mutually unbiased bases with fisher information," Phys. Rev. A 88, 052110 (2013).

${ }^{66}$ C. Spengler and B. Kraus, "Graph-state formalism for mutually unbiased bases," Phys. Rev. A 88, 052323 (2013).

${ }^{67}$ C. Godsil and A. Roy, "Equiangular lines, mutually unbiased bases, and spin models," European J. Combin. 30, 246-262 (2009).

${ }^{68}$ W. M. Kantor, "Mubs inequivalence and affine planes," J. Math. Phys. 53, 032204 (2012).

${ }^{69}$ H. F. Chau, "Unconditionally secure key distribution in higher dimensions by depolarization," IEEE Trans. Inf. Theory 51, 1451-1568 (2005).

${ }^{70} \mathrm{R}$. Gow, "Generation of mutually unbiased bases as powers of a unitary matrix in 2-power dimensions," arXiv:math/0703333v2 (2007).

${ }^{71}$ O. Kern, K. S. Ranade, and U. Seyfarth, "Complete sets of cyclic mutually unbiased bases in even prime-power dimensions," J. Phys. A 43, 275305 (2010).

${ }^{72}$ U. Seyfarth and K. S. Ranade, "Construction of mutually unbiased bases with cyclic symmetry for qubit systems," Phys. Rev. A 84, 042327 (2011).

${ }^{73}$ U. Seyfarth and K. S. Ranade, "Cyclic mutually unbiased bases, Fibonacci polynomials and Wiedemann's conjecture," J. Math. Phys. 53, 062201 (2012).

${ }^{74}$ V. E. Hoggart and M. Bicknell, "Roots of Fibonacci polynomials," Fibonacci Quart. 11, 271-274 (1973).

${ }^{75}$ J. L. Goldwasser, W. Klostermeyer, and H. Ware, "Fibonacci polynomials and parity 
domination in grid graphs." Graph. Combinator. 18, 271-283 (2002).

${ }^{76}$ R. Gow, "The equivalence of an invertible matrix to its transpose," Linear Multilinear A. 8, 329-336 (1980).

${ }^{77}$ D. Z. Djokovic, F. Szechtman, and K. Zhao, "An algorithm that carries a square matrix into its transpose by an involutory congruence transformation," Electron. J. Linear Al. 10, 320-340 (2003).

${ }^{78}$ V. C. Venkaiah and S. Sen, "Computing a matrix symmetrizer exactly using modified multiple modulus residue arithmetic," J. Comp. Appl. Math. 21, $27-40$ (1988). 